\title{
Democracia y ciudadania: un debate permanente para la construcción de la política pública de las mujeres*
}

\section{Democracy and citizenshipi: an ongoing debate for the construction of a public policy for women}

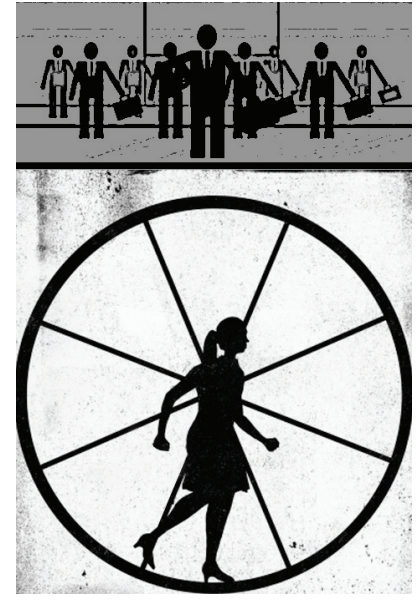

Eucaris Olaya**

Resúmen: Este texto forma parte de la reflexión y el debate entorno a los conceptos de democracia y ciudadanía desde una perspectiva feminista. El sistema económico capitalista y la lógica patriarcal son responsables de las profundas desigualdades, injusticias y opresiones en las que se encuentran millones de mujeres en el mundo. La lucha y resistencia feminista por el derecho a tener derechos se convierte en una dinámica tensa y contradictoria en escenarios que se afirman democráticos y que buscan incidir en la construcción de políticas públicas para las mujeres.

Palabras clave: Democracia, ciudadanía, igualdad, derechos humanos.

\begin{abstract}
This paper is part of the reflection and debate around the concepts of democracy and citizenship from a feminist perspective. The capitalist economic system and patriarchal logic are responsible for the vast inequalities, injustices and oppressions which are millions of women worldwide. The feminist struggle and resistance for the right to have rights becomes a tense and contradictory dynamics on places that call themselves democratics which aim to influence the construction of public policies for women.
\end{abstract}

Keywords: Democracy, citizenship, equality, human rights.

* Este artículo forma parte del marco histórico crítico de la Tesis de Doctorado en Trabajo Social: "Dinâmica na construção da Política Pública de Mulher e Gênero em Bogotá, 2004-2011".

** Doctora en Trabajo Social de la Pontifícia Universidade Católica de São Paulo. Docente de Trabajo Social de la Universidad de la Salle en Bogotá _ Colombia.E-mail: eolaya@unisalle.edu.co. 


\section{Presentación}

nalizar las desigualdades sociales en contextos que se afirman demo-
cráticos consiste en realizar un ejercicio crítico frente al significado de
los conceptos de democracia y ciudadanía. Las mujeres han conside-
rado que estos conceptos no se han puesto en práctica, y por el contrario, se han "naturalizado" las desigualdades e injusticias que se comenten contra gran parte de la humanidad.

El movimiento feminista ha interrogado los fundamentos que defiende la sociedad capitalista y patriarcal. Ha puesto en cuestión la igualdad, la justicia, y los derechos que serían garantizados en un escenario que se afirma democrático. Según Folguera, "Las mujeres están tomando un papel de liderazgo para redefinir conceptos tales como desarrollo, democracia, derechos humanos, seguridad internacional o medio ambiente" (2010, p. 91).

Un escenario que no garantiza la igualdad entre mujeres y hombres no se puede señalar como democrático. La desigualdad se refleja en millones de mujeres que mueren por efecto de la pobreza, de la violencia, como bien lo afirma Facio (1997, p. 351), "La desigualdad entre hombres y mujeres mata". La misma autora, indica que la desigualdad traspasa todos los ámbitos, incluyendo las relaciones de pareja:

Miles somos golpeadas y asesinadas por nuestros compañeros afectivos; las mujeres no tenemos el mismo poder dentro de las estructuras políticas, económicas, científicas y religiosas, miles morimos de desnutrición, en abortos clandestinos o prácticas culturales como la mutilación genital y en cirugías estéticas y obstétricas innecesarias. (Facio, 1997, p. 351)

El 8 de marzo de 2011, se reivindicaron los 100 años en que se celebra el día internacional de la mujer y los datos reflejan las profundas desigualdades entre mujeres y hombres: solamente 18 mujeres ejercen funciones de presidenta o primer ministra de un total de 192 Estados miembros de las Naciones Unidas; las mujeres representan el $70 \%$ de los pobres en el mundo, ellas realizan el $66 \%$ de todos los trabajos, y producen más de la mitad de los alimentos que consume la humanidad; sus ingresos no superan el 10\% de las ganancias y son dueñas solamente del 1\% de las propiedades del mundo ${ }^{1}$.

1. Tickner, Arlene. En "nuestro día”. En: El Espectador. Disponible en: <http://www.elespectador.com/ impreso/columna-255702-nuestro-dia>. Acceso en: 8 mar. 2011. 
Lo más grave en sistemas que se señalan "democráticos" es que las mujeres todavía no son reconocidas, ni valoradas como seres plenas de la especie humana. Las estructuras sociales tienen barreras sutiles que obstaculizan la participación femenina; y quienes mantienen la concentración del poder y la toma de decisiones en el mundo son los varones que representan clases privilegiadas y eminentemente conservadoras, impidiendo la construcción de sociedades justas, igualitarias, y realmente democráticas.

\section{La historia registra parcialmente las desigualdades}

La historia universal es una historia de guerras y dominación. "La historia que se nos transmite es una historia de las grandes batallas, una historia de las naciones en su lucha por conseguir realizar su destino, es una historia de origen; una historia de supervivencia, lucha y poder. Una fuerza representada por la superioridad masculina" (Miyares, 2003, p. 162).

Desde esta lectura, la humanidad mantiene una "deuda social con las mujeres" y la negación de los derechos de las mujeres es la negación de los derechos humanos en su sentido más amplio y profundo. Según Facio (1997), los derechos humanos son el producto de un periodo específico en la historia y de una región específica del mundo - el Renacimiento en Europa occidental - donde los valores que están en la base de lo que hoy consideramos como derechos humanos, son los que surgen de los valores filosóficos, políticos y religiosos dominantes del Renacimiento.

Los valores que le dan legalidad a los derechos humanos son producto de tensiones, contradicciones, fuerzas y acuerdos dentro de grupos que se constituyen en una sociedad. Sin embargo, son valores en lógicas androcéntricas, como bien lo han señalado los grupos feministas: "la Declaración de los Derechos del hombre - Derechos del ciudadano tiene una visión androcéntrica influenciada por ideas filosóficas del liberalismo individualista" (Facio, 1997, p. 354).

En las revoluciones estadounidense y francesa, el principio de igualdad era entendido como la igualdad de los hombres ante la ley, y específicamente hombres blancos, burgueses, letrados, con propiedades; por tanto, el concepto de igualdad no refería a toda la humanidad, excluía a los hombres pobres, iletrados, de grupos étnicos, y por supuesto, a las mujeres. 
Entre las dinámicas que se presentaban en el escenario de Europa occidental toman forma algunas de las propuestas que se venían gestando por las mujeres en diversos momentos de la historia. La primera vez que se planteó la cuestión de igualdad de género fue en la Revolución Inglesa a mediados del siglo XVII:

Los Diggers (Asociación de campesinos pobres) en 1646 se opusieron a la monogamia, promovieron la libertad sexual y exigieron la eliminación de la propiedad privada. Estas propuestas eran contrarias a las posiciones de la iglesia y las clases aristocráticas de la época. (Schaumberg, 2009, p. 52)

Posteriormente, durante la Revolución Francesa había un sentimiento igualitarista que fluía en el conjunto social, y las mujeres, que por su origen de clase privilegiada habían conseguido acceder a grados incluso amplios de cultura, lograron la vindicación de los Derechos de la mujer en 1792; sin embargo, sus ideas no pudieron llegar más allá que a un pequeño grupo de intelectuales (Valcárcel, 2008). El papel que jugó Olympe de Gouges con la Declaración de los Derechos de la Mujer y la Ciudadana, en 1791, fue un desafío al sistema que le costó su vida, y es guillotinada dos años después de haberla escrito. Este fue uno de los hechos más contradictorios, porque aunque fuera allí el escenario donde se defendían los valores de la libertad, la igualdad y la fraternidad, éste no incluía a las mujeres, y como se registra: "en uno de los proyectos de ley elaborado por un grupo radical de la Revolución, se proponía prohibir a las mujeres que aprendieran a leer" (Valcárcel, 2008, p. 65).

Mary Wollstonecraft, escritora y feminista inglesa, siguió parte del proceso y continuó reivindicando los derechos de las mujeres. Hizo énfasis sobre el papel del Estado, su responsabilidad por la igualdad entre hombres y mujeres, y la garantía de una educación universal y gratuita para ambos sexos.

Se podría afirmar que los postulados hechos por estas mujeres - y por otras que la Historia ha olvidado, o jamás reconoció — eran la exigibilidad de la igualdad de los sexos, el reconocimiento por parte del Estado frente a sus derechos y su ciudadanía. Para algunas, se necesitaban cambios legislativos y el derecho a voto, que se consideraba fundamental. Concomitantemente, existía un profundo debate frente a la condición de la mujer, sus diferencias en términos biológicos y especialmente la "facultad" de la maternidad como algo "natural" a su ser, como siendo la cuidadora de la familia.

Según Kappeli (1993, p. 193), "la misma interpretación diferente de la igualdad conduce a una división de las mismas mujeres en 'ciudadanas' y 'burguesas'. Tan 
pronto la cuestión feminista aparece como una cuestión político-legislativa, tan pronto como una cuestión ético-social". Por otra parte, esta tensión evidencia una lectura de intereses en una sociedad patriarcal. La misma autora Kappeli (1993, p. 204) señala que "las reivindicaciones jurídicas de las mujeres sólo tienen sentido cuando, al mismo tiempo, cuestionan las relaciones de poder en su conjunto".

Las primeras irrupciones hechas por mujeres al espacio público se han realizado en clubes, asociaciones y movimientos literarios, culturales, políticos y algunas eran disidentes religiosas, en Europa y Norteamérica entre los siglos XVIII y XIX. Como bien se conoce en la historia del Trabajo Social, "las organizaciones de caridad fundadas en Inglaterra y varios países de Europa se convirtieron en escenarios para que las mujeres se agruparan en torno a la filantropía y la caridad" (Perrot, 1993, p. 156).

Fundamentalmente, las mujeres burguesas se acercaron a otro mundo (lo público) que les permitió administrar recursos y hacer gestiones en beneficio de los pobres, así mismo, acceden a cumplir parcialmente funciones en la enseñanza, la asistencia, e intervienen en áreas de higiene, hábitat y atención hospitalaria. De otra parte, se realizaron los primeros ejercicios para divulgar sus ideas a través de periódicos y espacios políticos y literarios. Como bien lo señala Perrot: "En los límites de lo político y lo social, de lo público y lo privado, de lo religioso y lo moral, este crisol de identidad fue un auténtico laboratorio de experiencias para las mujeres" (1993, p. 162).

Entre las grandes conquistas de las feministas durante el siglo XIX, se consideran el derecho restringido, a la educación, al sufragio, a un salario y su vinculación a participar de procesos sindicales, políticos y comunitarios. Para algunas feministas radicales eran los primeros pasos hacia la igualdad de derechos, tanto en la vida privada como en la pública. Aunque estas conquistas no fueron generalizadas a todas las mujeres, como bien lo afirma Valcárcel (2008, p. 88), "A partir de 1880 algunas universidades europeas comenzaron a aceptar mujeres en sus aulas, siendo consideradas 'casos excepcionales', y las primeras mujeres que obtuvieron títulos encontraron negativas por parte de los colegios profesionales para que pudieran ejercer como médicas, juristas o profesoras".

Para el siglo XX, y después de la segunda guerra, prospera un ambiente de fuerzas democráticas; se cuestiona la opresión totalitaria y se hace más evidente la expresión de los derechos del individuo. De este "resplandor" se benefician en cierto sentido las mujeres. Como lo planteó Sineau (1993, p. 127), "la Declaración 
Universal de los Derechos del Hombre (1948) menciona la igualdad entre los sexos, así como la igualdad entre los esposos durante el matrimonio". Este insumo se hizo presente en las Constituciones completamente nuevas en países occidentales (Francia, Italia, Alemania), decidiéndose por incluir esta igualdad en relieve en sus respectivas leyes fundamentales. Sin embargo, la puesta en marcha de políticas y acciones que garantizaran la igualdad es un tema de debate permanente en los grupos feministas.

En los países latinoamericanos, donde por razones religiosas, políticas, económicas y culturales el feminismo llegará más tarde, necesitarán más tiempo para plantear el igualitarismo en la estructura social. Sólo a partir de los años setenta del siglo XX se producirán con notable velocidad las reformas relativas a los puntos esenciales de la libertad de las mujeres. Y esto ocurre en parte por las presiones de movimientos feministas ante organismos internacionales, entre ellos, la Organización de las Naciones Unidas, que sobresalieron su preocupación por la población femenina, especialmente, cuando se referían al papel de la mujer en el desarrollo social y económico de un país.

En la misma década de los setenta, la Asamblea General de las Naciones Unidas aprobó la proclamación de 1975 como el "Año Internacional de la Mujer" y la celebración de la primera Conferencia Mundial de la Mujer. También, se declaró la "Década de la Mujer" (1975-1985), cuyos objetivos se dirigían hacia la promoción de medidas encaminadas a integrar a las mujeres al desarrollo, crear condiciones de igualdad entre hombres y mujeres y contribuir al fortalecimiento de la paz mundial.

Sin embargo, "En la práctica, la prometida igualdad se limitó a meras palabras. Las mujeres son consideradas en las instituciones internacionales, en especial en las Naciones Unidas, simplemente como un área, un aspecto marginal de las políticas generales de las Naciones Unidas" (Folguera, 2005, p. 136). Se denuncia el énfasis de parte de la NU de balancear la representación ideológica y geográfica como modo de equilibrio de los intereses políticos y geoestratégicos, y como muestra de la democratización del sistema mismo, pero no se atiende a la democratización en clave paritaria. "Las mujeres quedan ausentes de los grandes temas que ocupan la agenda internacional y global, y a su vez, las cuestiones relativas a la igualdad y el género tampoco atraviesan dichos temas" (Maquieira, 2006, p. 56).

Retomando a Miyares (2003), todo relato histórico construye sus supuestos a partir de una teoría política que aporta al engranaje conceptual y no sólo describen 
los modelos de Estado y las formas de gobierno, sino también teorías de la historia. La teoría política ha sido alimentada por propuestas liberales basadas en la propiedad, $\mathrm{y}$ las libertades individuales que garantizan el poder y la riqueza. Fortaleciendo a un Estado que defiende las libertades individuales, se favorece la acumulación del capital, el libre mercado, y en palabras de Cortes: "los derechos conquistados en décadas anteriores pasaron a un proceso de reformas de parte del Estado para dar respuesta a las políticas neoliberales que benefician el libre mercado" (2006, p. 74).

\section{Las mujeres insisten en la democracia y en tener derechos}

La democracia se convierte en el ideal o tal vez en la utopía, y las mujeres le apuestan a la construcción de una sociedad democrática y pluralista. Sin embargo, son concientes que las dinámicas sociales, políticas, económicas y culturales en un sistema capitalista, patriarcal y guerrerista impiden la consolidación de un proyecto verdaderamente democrático. Son innumerables los debates entorno al significado de la democracia, a sus principios y al ejercicio de la ciudadanía, a la garantía plena de los derechos de mujeres, varones, jóvenes, niños y niñas.

El movimiento feminista desde sus orígenes se identificó con los valores de la democracia, con la idea que la democracia siempre debe estar presente, y sobre todo, que cumple un papel fundamental en el control de los ejercicios de poder. En la sociedad son fundamentales las formas organizativas a la hora de fijar objetivos, sin dejar de lado las subjetividades, la relación de lo público y lo privado como pilares fundamentales en la construcción de la democracia y la ciudadanía para las mujeres. Como lo dijo Phillips, "lo personal es político - constituyéndose en un lema del movimiento feminista, en el cual el poder era algo omnipresente resaltando la importancia de la democracia en todos los marcos de la vida humana" (1996, p. 79).

Pensar en el significado de "ciudadanía" como un ejercicio pleno en la toma de decisiones de una sociedad democrática exige de unos mínimos para todas y todos. Desde esta perspectiva, debe contener iguales y efectivas oportunidades para todas y todos en el marco de la política, todas las personas tendrán la oportunidad de votar y ser votados/as. También cada persona debe conocer las políticas y las consecuencias de éstas en sus vidas. Según Roth (2006), este ejercicio se convierte en la posibilidad de asumir compromisos ciudadanos, y serían las personas 
quienes indicarían los temas de la agenda política y siempre estarían abiertas para el debate y los cambios. Finalmente, todas las personas tendrían los derechos completos: información oportuna, efectiva participación, derecho a la igualdad de voto, a la oportunidad de acceder a la comprensión de los temas, y el derecho a participar ejerciendo el control sobre la agenda política.

Así mismo, se convierte en una relación de doble vía, más allá de la participación política en el ámbito democrático, se considera fundamental el papel que deben cumplir quienes gobiernan, pues tienen la responsabilidad de implementar políticas que garanticen los derechos humanos, pues éstos constituyen un interés público que todo sistema de gobierno democrático debe garantizar, preservar y extender a toda la población (Roth, 2006). No basta con promesas, se requiere que sean responsables por ponerlos en práctica, que tengan efectos concretos en la sociedad, que se materialicen en el bienestar y la calidad de vida de las personas, y en este caso de quienes la sociedad ha históricamente excluido.

Desde otra perspectiva, la democracia real presenta innumerables imperfecciones empezando por las instituciones políticas que están lejos de alcanzar un ideal de democracia, especialmente porque éstas deberían garantizar el ejercicio de una democracia representativa antes que una democracia directa. Como lo dijo Dahl (2004), la democracia representativa presupone que todos sus ciudadanos y ciudadanas adultos/as posean un cuerpo de derechos fundamentales, libertades, y oportunidades.

Para las feministas, estas reflexiones sobre la democracia representan diversos aspectos que los hombres no han tenido en cuenta. En primera instancia, hubo un período de insatisfacción generalizada por la propuesta de la democracia liberal, especialmente por las jerarquías y los dirigismos del patriarcado en el poder. Durante las décadas del sesenta y setenta, los grupos radicales le apostaron a una democracia más activa y participativa, esa democracia directa se reflejaba en salir del anonimato del voto, y compartir experiencias y conocimientos, llegar a niveles de asambleas y debates amplios y colectivos. "Las feministas se ocuparon de lo que podíamos llamar el micro nivel de la democracia en el seno de un movimiento y la democracia en la vida cotidiana" (Phillips, 1996, p. 80).

Es en dicho escenario que las feministas le apostaron a los valores de la democracia local y descentralizada. Posteriormente, y con algunos alcances, resurge la confianza en los mecanismos de la democracia liberal: "es posible llegar a niveles más altos y es posible tener poder”. A esto, Phillips (1996) comenta que la 
transición desde la participación hacia la ciudadanía refleja el movimiento en el pensamiento radical como conjunto. Esta etapa se caracteriza por la ocupación del macro nivel de la pertenencia de las mujeres a la comunidad política, a explorar cuestiones de inclusión en diversas esferas, y a amortiguar las pretensiones universalistas del pensamiento político moderno.

Entre esta participación y el movimiento político, las feministas evidencian y denuncian las debilidades que tiene la "promesa de igualdad". No existe igualdad para todas y todos; las mujeres no tienen los derechos que se denominan "universales" e "inalienables" y el camino que se traza el movimiento feminista es por la lucha democratizadora para que se amplie el reconocimiento de las mujeres, el derecho universal a tener derechos, como bien lo señaló Elizabeth Jelin. Las mujeres ante los derechos humanos es el desafío mayor, porque exige una participación activa en la redefinición permanente del concepto mismo de derechos humanos, su contextualización en las relaciones sociales, en las relaciones de género y en lógicas del poder hegemónico que ha impuesto el sistema capitalista (Maquieira, 2006, p. 51-52).

De otra parte, la forma de ejercer poder en un escenario donde impera la economía de mercado, donde la violación de los derechos civiles y políticos se facilita por la corrupción y la ausencia de gobernantes éticos, por la erosión de las instituciones estatales, por las debilidades y falta de garantías que la sociedad civil organizada tiene para exigir el cumplimiento del contrato social; todos estos aspectos impiden consolidar la democracia.

En palabras de Puyana (2004, p. 220), "Las políticas económicas, la guerra y otras problemáticas han incidido para que se agudicen las desigualdades sociales, afectando mayoritariamente a las mujeres", y se complementa con lo que dice Phillips (1996, p. 81): "Mujeres, nuestro desigual acceso a recursos económicos se combina con nuestro desigual acceso al conocimiento, a la información y a la formación política que nos hace políticamente (y no sólo socialmente) desiguales".

Phillips retoma a Dahl (1996, p. 81) y se pregunta: “¿la igualdad política es compatible con la economía de mercado?". Según el profesor brasileño, Francisco de Oliveira (2004, p. 115). "la política institucional gira en falso, pues los condicionamientos y constreñimientos impuestos por la globalización vuelven inútiles y superfluas las instituciones democráticas y republicanas". La ciudadanía está en una trampa porque está subordinada a la lógica capitalista y de mercado, y por tanto, renuncia a la política progresista y de derechos. 
Entre las trampas que ha impuesto el sistema está la defensa de las libertades, "somos libres, vivimos en democracia” y ¿qué ocurre con la igualdad y la justicia? Como bien lo indica Phillips (1996), en los planteamientos democráticos se habla de una igualdad que ignora o, de hecho, condona, las desigualdades sociales inherentes a la economía del mercado.

De otra parte, Vieira afirma: "La sociedad democrática es aquella en la cual ocurre una real participación de todos los individuos en los mecanismos de control de las decisiones, habiendo por tanto real participación de ellos y ellas en los rendimientos de la producción" (Vieira, 2007, p. 134). En su reflexión hace énfasis en la participación colectiva tanto en la toma de decisiones, como en las ganancias que se producen socialmente, dejando la condición de ser consumidores obedientes y aplicados al sistema económico.

La ciudadanía real, en una democracia real, solo se podrá lograr cuando las mujeres y las minorías poblacionales dejen de ser grupos oprimidos; en otras palabras:

Superar situaciones de explotación: cuando los beneficios derivados de su trabajo o energía van a otras personas sin que éstas les recompensen recíprocamente por dicho trabajo; de marginación: están excluidos/as de la participación en las principales actividades sociales, lo que en nuestra sociedad significa básicamente un lugar de trabajo con dignidad humana; falta de poder: viven y trabajan bajo imposiciones de otras personas que no garantizan dignidad; imperialismo cultural: como grupo están estereotipados/as y, a la vez, su experiencia y situación resulta invisible en el conjunto de la sociedad, por lo que tienen poca oportunidad y poca audiencia para expresar su experiencia y perspectiva sobre los sucesos sociales. (Young, 1996, p. 111)

Estos dos conceptos democracia y ciudadanía se deben relacionar con el reconocimiento de la igualdad. Sin embargo, para lograr la igualdad se debe partir por asumir la responsabilidad frente a las desigualdades que han consolidado históricamente y se han agudizado con el modelo económico imperante.

La democracia surgió justamente para responder a la necesidad de reconocimiento de las minorías [...]. Se debe entender la ciudadanía democrática como un conjunto de derechos y deberes válidos para todas y todos, aliado al concepto de igualdad, que no se restringe a la aritmética, no es mecánica, sino que se fundamentada en el reconocimiento de las profundas desigualdades que se han agudizado a través de los tiempos. (Benevides, 2004, p. 14) 
Sin embargo, las mujeres no son una minoría, por el contrario, representan la mitad de la humanidad. De alguna forma se observa y se confirma que quienes son los excluidos y excluidas del sistema son las mayorías: las mujeres, las y los pobres; sumado a las minorías en términos de grupos poblacionales como indígenas, negritudes, rom, entre otros. Esto evidencia las profundas desigualdades y la falta de garantías para el ejercicio pleno de la ciudadanía.

Es relevante el reconocimiento de los múltiples condicionamientos en los que la sociedad está inmersa, que en palabras de Paulo Freire, "somos seres condicionados pero no determinados", y desde los movimientos sociales existen propuestas democráticas y ciudadanas por la superación de la opresión, la injusticia y la desigualdad social. Como bien lo señaló Sposati $(2011$, p. 9) "el reconocimiento de lo que es universal para la población significa definir con lo que todos los seres humanos pueden contar" La sociedad democrática debe exigir la garantía del "derecho a tener derechos", el cumplimiento con unos maximin (Valcárcel, 2008) que garanticen la igualdad de oportunidades para todas y todos.

De otra parte, Benevides (2004) sugiere la importancia de fortalecer la democracia participativa, con fuertes mecanismos, como consejos populares, la participación administrativa en gestiones y en el diseño e implementación de normas de fiscalización y control, así como otras formas incipientes de democracia directa asociada a la democracia representativa, como iniciativa popular legislativa, en proyectos de ley. En ese sentido, las mujeres han incidido en el diseño de leyes y mecanismos de justicia, así como, en la formulación de políticas públicas para lograr el fortalecimiento de una sociedad democrática.

Se puede señalar que, salir de la opresión, es superar situaciones de violencia y muerte. Las mujeres reivindican la emancipación femenina como la opción de vida, de justicia social, de conquista permanente frente a los derechos, frente a la dignidad y el reconocimiento como humanas plenas.

\section{Las políticas públicas trascienden lo público y lo privado}

Indiscutiblemente, la lógica del sistema social de género asignó tareas y responsabilidades a las mujeres en función de su rol biológico centrado en la maternidad. Para autores como Muller (2009) en los siglos XVIII y XIX se "institucionalizó" la división social del trabajo, donde el cuidado familiar se ubica en el 
terreno de lo privado, y son las mujeres las que se encargan de cuidar y proteger a la especie, limitando su acción a la esfera reproductiva. Por otra parte, los hombres son los que se encargan de las actividades de producción que se desarrollan en el terreno de lo público, lo político, militar y económico, espacios valorados y reconocidos socialmente. El varón ejerce una función en la familia como proveedor. Esta división social repercutió gravemente en las condiciones de vida de las mujeres, y en las profundas brechas para participar en igualdad de condiciones en el terreno público.

Analizar cuidadosamente las condiciones de vida de las mujeres dedicadas al espacio doméstico familiar, y aquellas mujeres que se atrevieron, o se vieron en la necesidad de participar en el trabajo operario, o en actividades públicas, ha sido fuente de diversas investigaciones (Castells, 1996). Sin embargo, lo que más llama la atención, es que las mujeres a través del tiempo han asumido el trabajo productivo y han mantenido el trabajo reproductivo (asignado como "natural" a su género) duplicando su trabajo, tiempo y responsabilidades. Los estudios feministas lo han denominado las múltiples jornadas de trabajo femenino. Situación que no ha sido igual para los varones; la mayoría de ellos siguieron con su estatus de proveedor, y sin asumir mayores responsabilidades en el cuidado de la prole. En pleno siglo XXI, se mantiene la imagen que el hombre que participa de las labores domésticas familiares, está "ayudando", porque estas tareas son un deber que le corresponde a la mujer por "naturaleza" y se le considerada como la única responsable.

La teoría política feminista resalta que para superar la marginación y subordinación de las mujeres se requiere que la reflexión política se inicie abordando la esfera privada y se cuestione la supuesta "neutralidad" de los conceptos "universales" "naturales" que cargan el peso androcéntrico en lo político y en la forma de hacer política. Al respecto, la propuesta feminista, considera que para alcanzar la comprensión de la vida en su dimensión social, política, económica, cultural, ambiental, religiosa, entre otras, "es necesario aceptar que lo público y lo privado son dos caras de una misma moneda: el patriarcalismo liberal" (Paterman, 1996, p. 35).

Desde una lectura crítica, el Estado ha regulado las condiciones generales de producción y reproducción capitalista, comprendiendo en ellas la reproducción de la fuerza de trabajo masculina y femenina, el enfrentamiento de los conflictos y la administración de las crisis (Duque-Arrazola, 2006, p. 82). Por tanto, el Estado no es neutro, éste interviene en las relaciones sociales defendiendo intereses de clase, hegemónicamente patriarcal, androcéntrico, sexista y racista, lo cual se refleja en 
las Políticas públicas que tienen intencionalidades afectando diferencialmente a hombres y mujeres.

El Estado interviene directamente en las instituciones responsables de la socialización: la familia y la educación. Las acciones que implementa el Estado mantienen relaciones de poder y control en los espacios públicos y privados. Por lo tanto, "las mediaciones en las relaciones humanas no son neutras, y muchas de las acciones pueden contribuir para establecer acuerdos de justicia e igualdad, o por el contrario, legitimar la exclusión y la desigualdad social" (Miyares, 2003, p. 62).

Las feministas han enfatizado que lo personal es político, y al respecto, se observa cómo en circunstancias personales están presentes las decisiones públicas, mediadas por leyes y normas que reglamentan el cuerpo y las relaciones. En casos como la violación, el aborto, el estatus de soltera, esposa, separada, así como la orientación sexual, entre otras, se aplican leyes y se establecen políticas de acuerdo con el Contrato Social. En conclusión, los conflictos "personales" solamente se pueden solucionar por medios y acciones políticas.

De otra parte, desde la década de los años 1970 en el escenario latinoamericano, los programas y las políticas para las mujeres en el contexto latinoamericano partieron desde una perspectiva de bienestar liderada por las Naciones Unidas, en el que se buscaba institucionalizar un discurso sobre las mujeres y el desarrollo. Algunos gobiernos aceptan la inclusión de propuestas para las mujeres. Sin embargo, se mantienen unas premisas entorno a la maternidad considerado como el papel más importante; el cuidado y la crianza de los hijos como una tarea efectiva para el desarrollo, y se le asigna a la mujer un papel de receptora, consumidora y usuaria de servicios.

Sin embargo, era otro el debate que presentaban los movimientos de mujeres y feministas. El resplandor de los años setenta cuestiona "el modelo femenino" que mantenía y reproducía las condiciones desiguales entre mujeres y hombres. En el mundo occidental, la década de los setenta se caracterizó por la fuerza de las propuestas feministas y de la alianza con grupos de izquierda. Para Valcárcel (2008, p. 98), "el nicho político de nacimiento de la tercera ola del feminismo fue de la izquierda contracultural sesentaiochista".

Los fundamentos marxistas contribuyeron significativamente para la teoría política feminista. Se dio énfasis a temas de dominación del hombre por el hombre, y a la dependencia del orden de producción capitalista como el principal responsable de las desigualdades económicas, sociales, políticas, entre otras. El sistema 
reproducía el capital y explotaba a hombres y mujeres. A lo cual, las mujeres eran doblemente dominadas, tanto por el sistema económico, que no reconocía y — aún no reconoce su trabajo - , como el patriarcado, entendido este como "el orden socio moral y político que mantiene y perpetúa la jerarquía masculina. Un orden social, económico e ideológico que se autorreproduce por sus propias prácticas" (Valcárcel, 2008, p. 98).

En el contexto latinoamericano, el resplandor de la izquierda y su influencia en los movimientos sociales tuvo un impacto importante en los imaginarios sociales. Sin embargo, el papel que desempeñaron los Estados Unidos fue de combatir el "fenómeno de la izquierda" y la lucha anticomunista. Las posiciones conservadoras han defendido la propiedad, la familia y los valores morales cristianos, cuestionan las ideas libertarias y las propuestas defendidas por los grupos feministas y de izquierda.

Actualmente, la intromisión norteamericana no es ajena a las políticas internacionales, y las posiciones ortodoxas y conservadoras toman fuerza frente al "deber ser" de mujeres y hombres. Se defienden propuestas democráticas y participativas, pero la lógica de mercado se impone, agudizando la brecha social entre ricos y pobres. La ciudadanía de las mujeres en gran parte de los países del mundo se considera como una "concesión" y no como un derecho.

\section{Consideraciones finales}

De acuerdo con Lamus (2010), las mujeres latinoamericanas no han sido agentes pasivas de las políticas públicas, ni han estado supeditadas a los acuerdos con organismos internacionales, pues estos acuerdos no han sido en una única dirección, y por el contrario, han sido procesos complejos de negociación y concertación. Así mismo, las resistencias e incidencias políticas frente al Estado han sido estratégicas para superar (parcialmente) condiciones de subordinación que el sistema les ha impuesto a las mujeres. Por tanto, los movimientos de mujeres y feministas han sido concientes de las implicaciones y relaciones de poder que están en juego por la defensa y lucha de sus derechos.

Así mismo, las políticas, leyes, acuerdos, decretos, programas, no son suficientes en sí mismos. Se requiere de políticas que sean agentes de transformación cultural y de condiciones de vida. Las políticas para las mujeres no son neutras, ni 
están lejos de sus subjetividades, diversidades y conflictos. Las políticas deben contribuir a transformar las relaciones sociales marcadas por las profundas desigualdades, injusticias y opresiones. Los movimientos de mujeres y feministas deben continuar luchando por sus derechos, por sus ideales de libertad y justicia, así como defender identidades, diferencias y también des-identidades; en otras palabras, mantener propuestas emancipadoras, inclusive en el plano existencial, subversión de costumbres, relaciones sociales y estilos de vida.

Finalmente, el Estado tiene la responsabilidad de garantizar la participación ciudadana y contribuir al fortalecimiento de la democracia participativa. Los movimientos de mujeres y feministas se mantienen en defensa de una propuesta democrática pues se considera que cualquier totalitarismo, fanatismo o fundamentalismo refuerza el control social y normativo; limita el potencial humano e impide las garantías mínimas para la integridad del ser. "Sólo la democracia, en cuanto más profunda y participativa sea, asegura el ejercicio de las libertades y el goce de los derechos adquiridos. Por imperfecta que sea, es siempre mejor que la dictadura de cualquier naturaleza" (Valcárcel, 2008, p. 324).

Recebido em 10/11/2012 - Aprovado em 11/3/2013

\section{Referencias bibliográficas}

BENEVIDES, Maria Victoria. Mínimos de cidadania: ações afirmativas de enfrentamento a exclusão social. In: SPOSATI, Aldaíza (Coord.). Programa de Estudos Pós-Graduados em Serviço Social/PUC-SP, Núcleo de Seguridade e Assistência Social. São Paulo, 1994.

CASTELLS, Carme. Perspectivas feministas en teoría política. Barcelona: Paidós, 1996.

DAHL, Robert. A. Los sistemas políticos democráticos en los países avanzados: éxitos y desafíos. En: BORÓN, Atilio (Comp.). Nueva hegemonía mundial. Buenos Aires: Clacso, 2004.

DUQUE-ARRAZOLA, Laura. Política de assistência social e os tempos sociais femininos um caso brasileiro. Revista Nómadas, Bogotá, Instituto de Estudios Sociales Contemporáneos, Universidad Central, n. 24, 2006. 
FACIO, Alda. De las necesidades básicas a los derechos humanos. En: COOK, Rebeca. J. Derechos humanos de la mujer, perspectivas nacionales e internacionales. Bogotá: Profamilia, 1997.

FOLGUERA, Pilar. La equidad de género en el marco internacional y europeo. En: MAQUIEIRA, Virginia (Ed.). Mujeres, globalización y derechos humanos. Madrid: Feminismos, 2006.

FREIRE, Paulo. Política y educación. Buenos Aires: Siglo XXI, 1996.

KAPPELI, Anne-Marie. Escenarios del feminismo. En: DUBY, Georges; PERROT, Michelle (Comp.). Historia de las mujeres: siglo XIX, cuerpo, trabajo y modernidad. Madrid: Taurus, 1993.

LAMUS, Doris. De la subversión a la inclusión: movimientos de mujeres de la segunda ola en Colombia, 1975-2005. Bogotá, D.C. Instituto Colombiano de Antropología e Historia, 2010.

MAQUIEIRA, Virginia. Mujeres, globalización y derechos humanos. Madrid: Feminismos, 2006.

MIYARES, Alicia. Democracia feminista. Madrid: Feminismos/Cátedra, 2003.

MULLER, Pierre. Las políticas públicas. 3. ed. Bogotá, Universidad Externado de Colombia, 2009.

OLAYA, Eucaris. Dinâmica na construção da política pública de mulher e gênero em Bogotá. Tese (Doutorado em Serviço Social) — Pontifícia Universidade Católica de São Paulo, São Paulo, 2011.

OLIVEIRA, Francisco de. ¿Hay vías abiertas para América Latina? En: BORON, Atílio (Org.). Nueva hegemonía mundial. Buenos Aires: Clacso, 2004.

PATEMAN, Carole. Críticas feministas a la dicotomía público/privado. En: CASTELLS, Carme. Perspectivas feministas en teoría política. Barcelona: Paidós, 1996.

PERROT, Michelle. Salir. En: DUBY, Georges; PERROT, Michelle (Comp.). Historia de las mujeres: siglo XIX, cuerpo, trabajo y modernidad. Madrid: Taurus, 1993.

PHILLIPS, Anne. ¿Deben las feministas abandonar la democracia liberal? En: CASTELLS, Carme. Perspectivas feministas en teoría política. Barcelona: Paidós, 1996.

PUYANA, Yolanda. Política para las mujeres. Una constante paradoja. En: LAGUADO, D. Arturo. La política social desde la Constitución de 1991. ¿Una década perdida? Bogotá: Universidad Nacional. Ciencias Humanas, 2004. p. 219-36.

ROTH, D. André Noel. Discurso sin compromiso: la política pública de derechos humanos en Colombia. Bogotá: Aurora, 2006. 
SCHAUMBERG, Heike. La política generalizada en el siglo XXI perspectivas y desafíos. En: LAGARDE, Marcela. Liderar en clave de género: conferencias y foros virtuales. Quito: Centro Latinoamericano de Formación Política Mujeres y Ciudad, 2009.

SOARES, Vera. Políticas públicas para igualdade: papel do Estado e diretrizes. In: Políticas públicas e igualdade de gênero. São Paulo: Prefeitura de São Paulo. Coordenadoria Especial da Mulher, Secretaria do Governo Municipal, 2004.

SPOSATI, Aldaíza. O cidadão pode contar com o quê? Le Monde Diplomatique, São Paulo, ano 4, n. 43, fev. 2011.

SINEAU, Mariette. Las mujeres en la ciudad: derechos de las mujeres y democracia. En: DUBY, Georges; PERROT, Michelle (Comp.). Historia de las mujeres: siglo XX, la nueva mujer. Madrid: Taurus, 1993.

TICKNER, Arlene. En “nuestro dia”. El Espectador. Bogotá, 2011. Disponible en: <www. elespectador.com/impreso/columna-255702-nuestro-dia>. Acceso el: 8 mar. 2011.

VALCÁRCEL, Amelia. Feminismo en el mundo global. Madrid: Cátedra, 2008.

VIEIRA, Evaldo. Os direitos e a política social. 2. ed. São Paulo: Cortez, 2007.

YOUNG, Iris Marion. Vida política y diferencia de grupo: una crítica del ideal de ciudadanía universal. En: CASTELLS, Carme. Perspectivas feministas en teoría política. Barcelona: Paidós, 1996. 